\title{
NEW PROOF OF A THEOREM OF GAIFMAN AND HALES
}

\author{
BY ROBERT M. SOLOVAY
}

Communicated by D. Scott, November 16, 1965

For terminology on Boolean algebras see [2], [3]. In particular, a set $X$ generates a complete Boolean algebra $\Theta$ if $X \subseteq ß$, but for each proper complete subalgebra $B^{\prime}$ of $B$, we have $X \Phi B^{\prime}$.

1. Theorem 1 ([2], [3]). There are countably generated complete Boolean algebras of arbitrarily high cardinality.

Our proof, which is much simpler than those of Gaifman and Hales, is inspired by some recent work of Paul Cohen [1]. The connection between forcing and Boolean algebras will be elucidated in [5]. However, our proof will be independent of [1] and [5]. I am grateful to Dana Scott for helpful conversations concerning these proofs.

2. The example. Let $\boldsymbol{\aleph}_{\tau}$ be an infinite cardinal. We identify $\boldsymbol{\aleph}_{\tau}$ with the set of ordinals of cardinality less than $\boldsymbol{\aleph}_{\tau}$; we give $\boldsymbol{\aleph}_{\tau}$ the discrete topology. Let $X$ be the product space $\boldsymbol{\aleph}_{\tau}^{\aleph_{0}}$ endowed with the product topology. Thus an element $f \in X$ is a map from $\boldsymbol{\aleph}_{0}$ into $\boldsymbol{\aleph}_{\tau}$. Let $U(n, f)$ be

$$
\{g \in X: g(m)=f(m) \text { for } m \leqq n\} .
$$

The sets $U(n, f)$ for $n<\omega$ form a neighborhood basis of $f$.

Let $B$ be the algebra of regular open sets of $X$. (An open set $U$ is regular if $U=\operatorname{int}(\mathrm{cl} U)$ : Here int $E$ is the interior of $E$ and $\mathrm{cl} E$ is the closure of $E$. In particular, an open closed set is regular.) According to [4], $B$ is complete. Moreover, the Boolean operations $\Lambda, \vee, C$ (infinite sup, infinite inf, and complement) are given by the following formulas:

$$
\begin{aligned}
& \vee \mathfrak{u}=\operatorname{int}(\operatorname{cl} \cup \mathfrak{u}) \quad(\mathcal{U} \subseteq \beta) \\
& \wedge \mathfrak{u}=\operatorname{int}(\cap \mathfrak{u}) \quad(\phi \neq \mathfrak{U} \subseteq \mathbb{B}) \\
& C U=\operatorname{int}(X-U) \quad U \in \Theta \text {. }
\end{aligned}
$$

Using (2) and (3), one sees that $B$ is generated by the family $\left\{A_{n, \eta}: n<\omega, \eta<\boldsymbol{\aleph}_{\tau}\right\}$, where

$$
A_{n, \eta}=\{f \in X: f(n)=\eta\} .
$$

Moreover, if $\eta<\eta^{\prime}<\boldsymbol{\aleph}_{\tau}$, then the sets $A_{0, \eta}$ and $A_{0, \eta^{\prime}}$ are distinct. Thus the cardinality of $B$ is at least $\boldsymbol{\aleph}_{\tau}$. 
3. The generators. Let $B_{m, n}=\{f \in X: f(m) \leqq f(n)\}$. The sets $B_{m, n}$ are open closed, and thus determine elements of $B$. The following lemma will complete the proof of Theorem 1.

Lemma 1. The elements $\left\{B_{m, n} \mid m, n<\omega\right\}$ generate $B$.

Proof. Let $\mathbb{B}^{\prime} \subseteq \Re$ be the smallest complete subalgebra containing the elements $B_{m, n}$. Clearly $\{f \mid f(m)<f(n)\}$ lies in $\mathbb{B}^{\prime}$. (It has complement $B_{n, m}$.) To prove Lemma 1 , it suffices to show $A_{n, \eta} \in \mathbb{B}^{\prime}$ for every $n<\omega$, and $\eta<\boldsymbol{\aleph}_{r}$. (Since $\left\{A_{n, \eta}\right\}$ generates B.)

We prove this by induction on $\eta$. Assume then that for $m<\omega$, $\xi<\eta$, we have $A_{m, \xi} \in \mathbb{R}^{\prime}$. We shall show that the open closed sets $\{f \mid f(n)<\eta\}$ and $\{f \mid f(n) \leqq \eta\}$ lie in $\mathbb{B}^{\prime}$. By (3), (4) this will show that $A_{n, \eta} \in \mathbb{Q}^{\prime}$.

By (2),

$$
\{f \mid f(n)<\eta\}=\bigvee_{\xi<\eta} A_{n, \xi} .
$$

Thus $\{f \mid f(n)<\eta\}$ lies in $\mathbb{B}^{\prime}$. It follows that $C_{m}=\{f \mid f(m)<f(n) \rightarrow f(m)$ $<\eta\}$ lies in $\mathbb{B}^{\prime}$. To complete the proof, it suffices to show

$$
\{f \mid f(n) \leqq \eta\}=\bigwedge_{m \in \omega} C_{m} .
$$

In view of (2), it suffices to show

$$
g(n) \leqq \eta \leftrightarrow g \in \operatorname{int}\left(\bigcap_{m \in \omega} C_{m}\right) .
$$

The direction $\rightarrow$ of (7) is clear. Suppose now that $g(n)>\eta$, and $U(N, g) \subseteq \bigcap_{m \in \omega} C_{m}$. We shall get a contradiction. This will complete the proof of (7) and with that Lemma 1.

We may suppose that $N \geqq n$. Let $h: \boldsymbol{\aleph}_{0} \rightarrow \boldsymbol{\aleph}_{\tau}$ be defined as follows. For $m \leqq N, h(m)=g(m)$; for $m>N, h(m)=\eta$. Since $h \in U(N, g)$, we have $h \in C_{N+1}$. But this is absurd since

$$
\eta=h(N+1)<h(n)=g(n) \text { and } h(N+1) \geqq \eta .
$$

4. We sketch a proof of the following theorem. Let $\kappa$ be a regular cardinal.

Theorem $2([2],[3])$. There are complete $(\kappa, \infty)$ distributive Boolean algebras on $\kappa$ generators of arbitrarily high cardinality.

Proof. Let $X=\boldsymbol{\aleph}_{\tau}^{\kappa}$ endowed with the $\kappa$ topology. If $f \in X$, then $\{U(\alpha, f), \alpha<\kappa\}$ forms a neighborhood basis of $f ; U(\alpha, f)=\{g \in X \mid g(\beta)$ $=f(\beta)$ for $\beta \leqq \alpha\}$. Let $\beta$ be the complete Boolean algebra of regular open subsets of $X$. The proof of [4] Lemma 3, shows that $\beta$ is $(\kappa, \infty)$ 
distributive. (In Scott's terminology, $\beta$ is $(\gamma, \delta)$ distributive if $\gamma<k$ and $\delta$ is a cardinal.) Exactly as in the proof of Theorem 1, one sees that $B$ has cardinality at least $\aleph_{\tau}$, and that $B$ is generated by sets of the form

$$
\begin{gathered}
\left\{f \mid f(\eta) \leqq f\left(\eta^{\prime}\right)\right\}, \text { where } \eta, \eta^{\prime}<\kappa . \\
\text { REFERENCES }
\end{gathered}
$$

1. P. J. Cohen, The independence of the continuum hypothesis, Proc. Nat. Acad. Sci. U.S.A. 50 (1963), 1143-1158; II, ibid. 51 (1964), 105-110.

2. H. Gaifman, Infinite Boolean polynomials. I, Fund. Math. 54 (1964), 230-250.

3. A. W. Hales, On the non-existence of free complete Boolean algebras, Fund. Math. 54 (1964), 45-66.

4. D. Scott, The independence of certain distributive laws in Boolean algebras, Trans. Amer. Math. Soc. 84 (1957), 258-261.

5. R. Solovay, Boolean algebras and forcing, (to appear.)

University of California, Berkeley 